\title{
Die moderne evidenzbasierte psychiatrische Versorgung: Welchen Weg nimmt die institutionelle Schweizer Psychiatrie?
}

\author{
H. D. Brenner, W. Rössler, U. Fromm
}

\section{Einleitung}

Die Weiterentwicklung der institutionellen psychiatrischen Versorgung in den neunziger Jahren war in den meisten westlichen Industrieländern durch eine Fortführung der Schwerpunktverlagerung der Behandlung und Betreuung weg von den stationären hin zu ambulanten und teilstationären Hilfsangeboten geprägt. Neben den bereits früher propagierten Prinzipien der Normalisierung, Bedarfsorientierung und Gemeindeintegration («least restrictive alternative») gewannen Prinzipien wie Patientenzentrierung statt Institutionszentrierung, Ambulantisierung und integrierte Behandlung (Überwindung der Trennung zwischen ambulanter, teilstationärer und stationärer Behandlung) zunehmendes Gewicht und Bedeutung. Man kann in diesem Zusammenhang von einer eigentlichen zweiten Runde der Psychiatriereform sprechen. In der Schweiz folgte man dieser Entwicklung bisher nur zaghaft, viel eher stagnierte die Entwicklung während des letzten Jahrzehnts vielerorts. So gilt in der institutionellen Psychiatrie in der Schweiz entgegen dem allgemein anerkannten Prinzip «ambulant vor stationär» in der Realität immer noch «stationär vor ambulant». Traditionell bedeutet «ambulant» wenig, «stationär» viel Hilfe. Wer mit wenig Hilfe nicht auskommt, muss sich hierzulande also in stationäre Behandlung begeben, um viel Hilfe zu erhalten, auch wenn er davon nur einen Teil des stationären Behandlungsangebotes benötigt und zudem durchaus in seiner eigenen Wohnung und in seinem eigenen Bett verbleiben könnte. Dieser andauernde faktische Vorrang stationärer vor ambulanter Hilfe führt in nicht unerheblichem Umfang zu einem strukturbedingten Mehreinsatz von Ressourcen in Form institutionsbezogener Hilfspakete. Ein Aspekt, der nicht nur im Bereich der Psychiatrie, in jüngerer Zeit an der überproportionalen Kostensteigerung für durch stationäre Anbieter erbrachte ambulante Dienstleistungen deutlich wird. Diese Ressourcen könnten zum Teil sinnvoller, effektiver und sparsamer eingesetzt werden, wenn sie im Ausmass jeweils in flexibler
Kombination bedarfsorientiert und personenzentriert im natürlichen Lebensumfeld der Patienten zum Einsatz kämen. Es ist ein unhaltbarer Zustand, dass die wissenschaftlich gebotene Fortentwicklung des institutionellen psychiatrischen Versorgungssystems mangels entsprechender psychiatrischer Zielvorgaben in vielen Kantonen sowie mangels fehlender finanzieller Anreizsysteme $\mathrm{zu}$ einer versorgungspolitischen Umsteuerung nicht nur nicht mitvollzogen werden kann, sondern effektiv behindert wird. Soll der Anschluss an die Psychiatriereform in den umliegenden Ländern nicht verlorengehen, ist hier ein rasches Umdenken dringend erforderlich.

Nicht ganz überraschend war entsprechend die Entwicklung der institutionellen psychiatrischen Versorgung in der Schweiz durch eine steile Zunahme stationärer Aufnahmen geprägt. So hat sich schweizweit in den letzten 10 Jahren die Zahl stationärer Behandlungen etwa verdoppelt. Diese Entwicklung hat in den Medien grosse Aufmerksamkeit gefunden. Insbesondere wurde die Frage aufgeworfen, ob dieser Zuwachs stationärer Aufnahmen auf einen entsprechenden Zuwachs an psychischen Erkrankungen in der Schweiz zurückzuführen ist, und durch welche versorgungspolitischen Massnahmen die damit verbundenen Anforderungen am besten bewältigt werden können.

$\mathrm{Ob}$ psychische Erkrankungen tatsächlich häufiger werden, lässt sich gegenwärtig auf empirischer Basis nicht belegen. Der in den Medien vermittelte Eindruck eines vermeintlich starken Anstiegs steht vermutlich zum Teil mit der Psychologisierung von Alltagsproblemen, vor allem aber mit dem Wissenszuwachs und den damit verbundenen besseren Erkennungsmöglichkeiten sowie mit gesellschaftlichen Veränderungen insbesondere im Bereich der Familien und des Arbeitslebens in Zusammenhang. Eine echte Zunahme psychischer Störungen in der Bevölkerung resultiert jedoch sicher aus einer sich ändernden demographischen Zusammensetzung der Bevölkerung. So lässt z. B. die zunehmende Alterung der Bevölkerung die Zahl alterspsychiatrischer Erkrankungen wesentlich 
anwachsen. Recht gut belegt sind auch die Zusammenhänge zwischen den sozioökonomischen Rahmenbedingungen einer Gesellschaft und der Inanspruchnahme stationärer Leistungen. Ein solcher Zusammenhang zeigte sich beispielsweise auch bei einer Analyse der Inanspruchnahmedaten des Kantons Zürich. Hier weisen insbesondere städtische Quartiere mit höherem Ausländeranteil - Indikator für schwierigere soziale Lebensverhältnisse - erhöhte Raten stationärer Behandlungen auf.

Die Nutzung psychiatrischer Versorgungseinrichtungen wird zudem durch die räumlichzeitliche Entfernung beeinflusst, die zur Inanspruchnahme einer Versorgungsinstitution zu bewältigen ist. Personen mit psychischen Störungen sind nur eingeschränkt bereit oder fähig, lange Anreisezeiten zu bewältigen. Anreisezeiten von mehr als einer halben Stunde können die Krankenhaushäufigkeit in psychiatrischen Kliniken bis um die Hälfte reduzieren. Dies ist ein Befund, der im Hinblick auf die geographischen Besonderheiten der Schweiz besonderer Beachtung bedarf und auf einen gegenwärtig bestehenden Strukturmangel hinweist. Dabei sind es vor allem schwierig zu behandelnde Patienten, etwa mit Störungen durch primären oder begleitenden Substanzmissbrauch, die eine mangelnde Behandlungsmotivation aufweisen. Diese $\mathrm{Zu}-$ sammenhänge gelten mit einiger Wahrscheinlichkeit auch für die psychiatrische Regelversorgung der Schweiz. Wenn z. B. die psychiatrischen Versorgungsaufgaben durch Vertragskliniken ausserhalb des Kantons übernommen werden, kommt dieser Einfluss der Entfernung deutlich zum Tragen. Dies gilt z.B. für die Kantone Zug, Uri und Schwyz, die gemeinsam durch die Klinik Oberwil in Zug versorgt werden. In Uri und Schwyz liegt die Häufigkeit einer psychiatrischen Krankenhausbehandlung um jeweils mehr als ein Drittel niedriger als in Zug.

Auch Einflussvariablen auf seiten der Leistungsanbieter sollten nicht vernachlässigt werden. Beispielsweise findet sich eine beachtliche Variationsbreite in der Zahl psychiatrischer Klinikpflegetage pro 1000 Einwohner über verschiedene Kantone hinweg. So weist Zürich beinahe doppelt so viel Pflegetage auf wie Genf. Solche Unterschiede lassen sich kaum durch eine höhere Prävalenz oder Inzidenzrate der gegenwärtig bedeutsamsten psychiatrischen Erkrankungen oder durch besondere inanspruchnahmefördernde Faktoren erklären. Es ist zu vermuten, dass hinter dieser Beobachtung unterschiedliche Behandlungsphilosophien und im besonderen eine unterschiedliche Struktur der institutionellen psychiatrischen Versorgungs- angebote mit stärkerer Gewichtung der gemeindeintegrierten Hilfen stehen.

In diesem Zusammenhang sollten zudem einige Besonderheiten der psychiatrischen Versorgung in der Schweiz im europäischen Vergleich beachtet werden. Mit einem niedergelassenen Psychiater auf etwa 5000 Einwohner verfügt die Schweiz über eine mehr als zweimal höhere Niederlassungsdichte als das ebenfalls im internationalen Vergleich gut ausgestattete Deutschland. Im Durchschnitt verfügen die Nachbarländer der Schweiz mit 0,6-0,8 Betten pro 1000 Einwohnern auch nur über die Hälfte der in der Schweiz aufgestellten psychiatrischen Betten (1,4 Betten pro 1000 Einwohner). Wenn also trotz dieses nachweisbar hohen Ausbaustandards des stationären Versorgungsschenkels der Aufnahmedruck gerade auf diese stationären Einrichtungen enorm angewachsen ist, kann dem sicherlich nicht mit der Schaffung neuer (Akut-)Betten - wie kürzlich in einigen Kantonen geschehen - wirksam begegnet werden. Die Gründe für den in der Schweiz aktuell beobachteten Aufnahmedruck auf den stationären Versorgungsbereich sind durchwegs komplex und bedürfen im einzelnen gegenwärtig noch einer genaueren Analyse. Im vorliegenden Rahmen können daher nur einige der relevant erscheinenden Aspekte facettenartig skizziert werden.

Einmal ist hier ein verändertes Zuweisungsund Weiterweisungsverhalten beteiligter Institutionen zu sehen. Wenn wir davon ausgehen, dass 25 bis 30 Prozent der Klientel in der Allgemeinpraxis wegen psychischer Störungen behandlungsbedürftig sind, wie dies jüngste Studien der WHO gezeigt haben, ist es selbstverständlich, dass nur ein kleiner Teil davon in spezialisierten psychiatrischen Institutionen behandelt wird. Der überwiegende Teil psychisch Kranker wird üblicherweise in ambulanten und stationären Einrichtungen anderer medizinischer Disziplinen behandelt. Kommt es aus verschiedenen Gründen - z.B. der gesundheitspolitisch geforderten Verkürzung der Verweildauer in Allgemeinspitälern - zu Änderungen in der Aufnahme- und Entlassungspolitik oder der Überweisungspraxis, kann dies schnell zu einem bedeutsamen Zuwachs der Patientenzahlen in psychiatrischen Kliniken führen.

Möglicherweise stehen die steigenden Zahlen stationärer Patienten auch mit einem Einstellungswandel der Bevölkerung gegenüber der Psychiatrie und psychisch Kranken in Zusammenhang. Ergebnisse einer eigenen Repräsentativbefragung der Schweizer Bevölkerung zeigen jedoch, dass bei psychischen Problemen Psychiater nicht die erste Anlaufstelle darstellen. Auch 
die von Psychiatern eingesetzten Medikamente stossen bei der Bevölkerung ganz überwiegend auf Ablehnung. Die höchste Akzeptanz im Gesundheitswesen geniessen hingegen Hausärzte. Diesen Hausärzten wiederum stehen häufig nicht genügend differenzierte Informationen über geeignete, zur stationären Behandlung komplementäre Versorgungsangebote (soweit vorhanden) zur Verfügung, um die gegenwärtig meist prioritäre Erwägung stationärer Einrichtungen zu hinterfragen.

Nicht zuletzt ist unter dem Eindruck eines veränderten politischen und sozialen Umfeldes in den vergangenen Jahren auch von politischer Seite zunehmend das Bedürfnis nach neuen Leistungsangeboten (etwa die psychologische Erste Hilfe im Bereich der Katastrophenmedizin) an die Psychiatrie herangetragen worden, ohne dass entsprechende Ressourcen in ausreichendem Mass bereitgestellt wurden. Dies hatte zur Folge, dass derartige neue Aufgaben wiederum mit Priorität den grösseren stationären Zentren angetragen wurden.

Schliesslich bleibt noch die gegenwärtige Tarifstruktur anzuführen, die in den meisten Bereichen der Schweizer psychiatrischen Versorgungslandschaft zu krassen Ungunsten nichtstationärer Versorgungsbereiche ausgestaltet ist und unter dem ständigen Druck wiederholter Sparrunden der letzten Jahre eine Umverlagerung von Ressourcen aus dem stationären in den ambulanten und teilstationären Versorgungsbereich oft verunmöglicht, da aus Sicht der stationären Träger eine solche Verlagerung immer mit dem Risiko einer globalen Kürzung verbunden ist.

Bei Betrachtung dieser Rahmenbedingungen muss der zukünftige Weg der Psychiatrie in der Schweiz sorgfältig geplant werden. Wie schon gesagt ist es schwer vorstellbar, dass dem wachsenden Aufnahmedruck in der Psychiatrie mit einer Erhöhung der Zahl psychiatrischer Betten wirksam begegnet werden kann. Wir werden für die Zukunft sorgfältig überlegen müssen, wo wir stehen und welche Entwicklung die psychiatrische Versorgung nehmen soll. Dies wird nicht selten mit der Frage verbunden sein, wo und wie sich die ständig knapper werdenden Mittel am effizientesten im Sinne einer qualitativ hochstehenden psychiatrischen Versorgung auf Ebene der zu versorgenden Bevölkerung einsetzen lassen. Bei diesen Überlegungen müssen wir verstärkt die internationalen Entwicklungstrends zur Kenntnis nehmen und im besonderen berücksichtigen, dass sich in den letzten zwei Jahrzehnten die therapeutischen Möglichkeiten enorm erweitert haben. Nicht nur die gemeindeintegrierte psychiatrische Rehabilitation hat sich zu einer evidenzgestützten empirischen medizinischen Disziplin entwickelt, wir verfügen heute darüber hinaus über hinreichende empirische Belege, dass es wirksame Möglichkeiten in der akutpsychiatrischen Behandlung im ambulanten Setting gibt. Dies soll im folgenden nach einem kurzen Rückblick auf den bisherigen Verlauf der Psychiatriereform im einzelnen dargestellt werden.

\section{Entwicklung der Psychiatriereform}

Zwischen 1950 und 1960 ging von den Missständen in der damaligen Krankenhauspsychiatrie, einer wachsenden Bedeutung der Rehabilitation nach dem zweiten Weltkrieg sowie der Entdeckung der Neuroleptika in mehreren westlichen Industrieländern der Anstoss zu den häufig unter dem Begriff der Deinstitutionalisierung zusammengefassten psychiatrischen Versorgungsreformen aus. Diese unterschieden sich in den einzelnen Ländern hinsichtlich ihres Beginns, ihrer Ausgangssituation und ihrer politischen Begründung, waren aber in ihrem weiteren Verlauf durch einige gemeinsame Entwicklungsleitlinien charakterisiert:

- Das psychiatrische Versorgungssystem sollte bedarfsgerecht und patientenzentriert sein, d.h., allen Behandlungsbedürftigen sollte entsprechend ihren Bedürfnissen die von den Erfolgsaussichten und dem Kostenaufwand her günstige Therapie ohne Zugangsbarrieren zuteil werden.

- Die psychiatrisch Kranken sollten den körperlich Kranken in rechtlicher, finanzieller und sozialer Hinsicht gleichgestellt und die psychiatrische Versorgung sollte in das bestehende System der allgemeinen Gesundheitsversorgung integriert werden.

- Die psychiatrischen Dienstleistungen sollten normalisiert und gemeindenah organisiert werden, d.h., psychisch Kranke sollten zu Behandlungszwecken nur so kurz wie unbedingt nötig aus Familie, Gemeinschaft und Arbeit herausgenommen werden, um ihnen die Wiedereingliederung zu erleichtern.

- Die verschiedenen Versorgungsangebote sollten koordiniert werden, um die Kontinuität der Behandlung zu gewährleisten und Fehlplazierungen sowie Mehrfachbetreuung zu vermeiden.

Mit diesen Versorgungsgrundsätzen untrennbar verbunden war das Primat der ambulanten und teilstationären gegenüber der vollstationären psychiatrischen Behandlung, die nur noch so 
selten und so kurz als möglich erfolgen sollte. Die Zielrichtung der Reformen war daher im wesentlichen durch den Aufbau bedarfsgerechter gemeindenaher Behandlungsstrukturen charakterisiert und beinhaltete im Kern einen Übergang von der klinikzentrierten zur gemeindeintegrierten Versorgung auch schwer und chronisch psychisch erkrankter Menschen.

Entsprechend ist seither eine Reihe von Modellen zur gemeindepsychiatrischen Versorgung entworfen worden. Dabei haben sich im deutschsprachigen Raum vor allem Ansätze etabliert, die auf der teilstationären Behandlung in Tageskliniken oder auf der Behandlung und Betreuung in Ambulatorien basieren. Im englischen Sprachraum dagegen herrschen heute Ansätze vor, die sich entweder am sogenannten «Assertive Community Treatment»-Modell (ACT) oder am Konzept des «Klinischen Case-Managements» orientieren [1-3]. Beide Ansätze sehen im Kern eine nachgehende und aufsuchende Behandlung von schwer psychisch Kranken mit 24stündiger Ansprechbarkeit des therapeutischen Teams sowie direkten Hilfeleistungen auch bei der Bewältigung praktischer Alltagsanforderungen im Gemeindeumfeld der Patienten vor. Die Behandlung wird entweder durch mobile Teams in gemeinsamer Verantwortung (ACT) oder durch eine feste Bezugsperson des Gemeindeteams (Case-Manager) sichergestellt. Das Gemeindeteam entscheidet über eine allfällige Überweisung in stationäre Behandlung und behält im Idealfall auch während des Klinikaufenthalts die Verantwortung. Der Wechsel von der Institutionszentrierung zur Patientenzentrierung wird damit bei diesen Ansätzen noch konsequenter vollzogen als in der tagesklinischen Behandlung oder der Behandlung und Betreuung in einem Ambulatorium.

\section{Gemeindeintegrierte Akutbehandlung}

Mit der primären Zielsetzung, stationäre Langzeitpatienten wieder in ihr Lebensumfeld einzugliedern, konzentrierte sich die Gemeindepsychiatrie zunächst überwiegend auf ihre Funktionen der Nachbetreuung und Rehabilitation. Insbesondere in den letzten zehn Jahren wurden jedoch vermehrt auch die Konzeption und Evaluation gemeindepsychiatrischer Ansätze zur Akutbehandlung und Krisenintervention vorangetrieben. Die Entwicklung konzentrierte sich dabei vor allem auf die Akutbehandlung im häuslichen Umfeld der Patienten sowie auf die Behandlung akuter Krankheitsepisoden in einer Tagesklinik.
Die Effektivität dieser beiden Ansätze ist in einer Serie kontrollierter Studien mit der Wirksamkeit vollstationärer Akutbehandlung verglichen worden (Übersicht bei [4]). Im Hinblick auf eine Verringerung der psychopathologischen Beeinträchtigungen oder auf eine Verbesserung der psychosozialen Anpassungsleistungen ergaben sich dabei meist keine signifikanten Wirkunterschiede zwischen gemeindeintegrierter und vollstationärer Akutbehandlung. Auch Rezidive fanden sich unter beiden Bedingungen im wesentlichen gleich häufig. Deutlich zugunsten einer gemeindeintegrierten Akutbehandlung fielen jedoch die Vergleiche des stationären Behandlungsbedarfs aus [5-11]. Die Behandlung akuter Krankheitsepisoden im Wohnumfeld der Patienten oder in einer Tagesklinik führte nämlich regelmässig zu einer erheblichen Verkürzung der Klinikaufenthaltsdauer, dies sowohl während der akuten Indexepisode als auch innerhalb des jeweiligen Katamnesezeitraumes. Darüber hinaus wurden die Angehörigen durch die gemeindeintegrierte Akutbehandlung nicht stärker belastet. Im Gegenteil - sie zogen die gemeindenahe Behandlung des erkrankten Familienmitglieds der Klinikbehandlung eindeutig vor, ebenso wie auch die Patienten selbst. Die einzelnen Untersuchungen lieferten zudem Hinweise für eine durch das gemeindeintegrierte Versorgungssetting erhöhte Therapiecompliance und Behandlungskontinuität.

Entscheidend für die Implementierung innovativer Behandlungsansätze ist im gegenwärtigen gesundheitsökonomischen Umfeld aber nicht nur ihr klinischer Nutzen, sondern auch ihr Kosteneffektivitätsverhältnis im Vergleich mit der Standardversorgung. Dies gilt für gemeindeintegrierte Ansätze zur psychiatrischen Akutbehandlung um so mehr, als Bemühungen um eine Verbesserung der Kosten-Nutzen-Relation eine der wesentlichsten Triebfedern für ihre Entwicklung waren. Entsprechend sind sie auch im Hinblick auf ihre Kostenwirksamkeit evaluiert worden. Die Ergebnisse dieser Kosteneffektivitätsstudien zeigten übereinstimmend, dass sich mit gemeindeintegrierten Behandlungsansätzen eine deutliche Senkung der direkten Kosten erzielen lässt [12-14]. Diese Kostensenkung war zum grössten Teil auf die niedrigeren stationären Behandlungskosten zurückzuführen. Die entsprechenden Einsparungen resultierten jedoch nicht nur aus der signifikant niedrigeren Klinikverweildauer, sondern auch aus den erheblich geringeren infrastrukturellen Kosten [15]. Die Verlagerung der Behandlung ins Haus der Patienten führte im weiteren weder zu einer Zunahme der finanziellen Belastung der 
Patienten selbst oder ihrer Angehörigen noch zu einem Kostenanstieg bei anderen lokalen Versorgungseinrichtungen. Auch bezüglich der indirekten Kosten infolge von Produktivitätsausfällen fanden sich keine Unterschiede zwischen den beiden Behandlungsansätzen. Allerdings wiesen die Ergebnisse der Kostenvergleichsstudien gleichzeitig darauf hin, dass eine gemeindeintegrierte Behandlung akuter Krankheitsepisoden nicht bei allen Patienten eine kostengünstige Alternative darstellt (z. B. [16]). So fiel die Senkung der Behandlungskosten bei psychotischen Patienten beispielsweise geringer aus als bei Patienten mit nicht psychotischen affektiven Störungen oder Doppeldiagnosen [17], und bei einem variablen Anteil aller Patientengruppen blieb kurzzeitig auch eine vollstationäre Behandlung erforderlich. Für eine kleinere Gruppe von Patienten kam die Behandlung im Wohnumfeld oder in einer Tagesklinik sogar teurer zu stehen.

Im Zusammenhang mit gemeindeintegrierten Ansätzen zur Behandlung akuter Krankheitsepisoden ist oft von möglichen negativen Auswirkungen die Rede. So wird befürchtet, dass es häufiger zu Suiziden kommt, die Mortalität aufgrund somatischer gesundheitlicher Probleme ansteigt oder die Patienten einem erhöhten Rückfallrisiko ausgesetzt werden, da sie möglicherweise in einem belastenden sozialen Umfeld belassen werden [18]. Für keine dieser Befürchtungen konnten in den vorliegenden Untersuchungen Anhaltspunkte gefunden werden. Auch die Befürchtung, dass eine gemeindeintegrierte Akutbehandlung zu einer Erhöhung der Obdachlosenrate führen könnte, hat sich empirisch nicht bestätigt. Ebenso wenig scheint - zumindest in Versorgungssektoren mit gemeindeintegrierter Akutbehandlung und intensiver Nachbetreuungdie Gefahr einer Vergrösserung der Anzahl unbefriedigter Therapiebedürfnisse oder einer Fragmentierung der Behandlung gegeben.

Insgesamt betrachtet lassen die Befunde der Wirksamkeits- und Kosteneffektivitätsstudien den Schluss zu, dass eine gemeindeintegrierte Behandlung akuter psychischer Krankheitsepisoden bei einem beträchtlichen Anteil aller heute zur stationären Aufnahme zugewiesenen Patienten mindestens gleich effektiv ist wie die Klinikbehandlung. Nach jüngeren und methodisch genaueren Schätzungen liegt dieser Anteil bei etwa 40 Prozent (siehe [19]). Durch die Integration gemeindeintegrierter Einrichtungen und Ansätze zur Behandlung akuter Krankheitsepisoden kann daher die Verringerung kostenintensiver psychiatrischer Klinikbetten weiter vorangetrieben werden. Gleichzeitig ist jedoch festzuhalten, dass die Rückgriffsmöglichkeit auf eine Behandlung in der Klinik erforderlich bleibt. In keiner der einschlägigen Untersuchungen konnte gänzlich auf die stationäre Behandlung verzichtet werden, was bedeutet, dass gemeindepsychiatrische Behandlungsansätze die Klinik nur zum Teil ersetzen können. Sie üben vielmehr eine zu ihr komplementäre Funktion in der psychiatrischen Regelversorgung aus. De facto wurden denn auch nicht ausschliesslich gemeindepsychiatrische mit rein stationären Behandlungsbedingungen verglichen, sondern jeweils verschiedene Versorgungssysteme mit mehr oder weniger gut ausgebauten gemeindepsychiatrischen Behandlungsstrukturen. Unklar bleibt vorerst noch, welche therapeutischen Komponenten in einem gemeindeintegrierten Behandlungssetting im Unterschied zur Klinikbehandlung wirksam werden. In den Studienergebnissen finden sich Hinweise darauf, dass die Triagefunktion des Gemeindeteams, die Stärkung der therapeutischen Allianz mit nicht professionellen Betreuern sowie die Verbesserung der Kooperation mit anderen Gesundheits- und Sozialhilfeeinrichtungen spezifische Wirkprinzipien der gemeindeintegrierten Akutbehandlung darstellen (vgl. [20]). Im weiteren zeigte sich, dass die gemeindeintegrierte Akutbehandlung die Eigenverantwortung der Patienten, ihre Teilnahme an therapeutischen und rehabilitativen Programmen sowie den Kontakt zu anderen Gesundheits- und Sozialhilfeeinrichtungen fördert. Neben den therapeutisch aktiven Wirkfaktoren gilt es in Zukunft auch, die Möglichkeiten und Grenzen einer effizienten Synchronisation der verschiedenen Aufgaben eines gemeindeintegrierten Versorgungssystems abzuklären [21].

\section{Gemeindeintegrierte Rehabilitation}

In den vergangenen zwei Jahrzehnten hat sich international gesehen auch die gemeindeintegrierte psychiatrische Rehabilitation zu einem effizienten und zeitgemässen Weg der Wiedereingliederung chronisch psychisch Kranker in die Gesellschaft entwickelt. In Übereinstimmung mit dem klassischen Rehabilitationsansatz, die Umwelt entsprechend den reduzierten Fähigkeiten und Möglichkeiten der Betroffenen zu gestalten, ist zunächst auch in der Schweiz in den letzten Jahrzehnten ein Netz geschützter Wohn- und Arbeitsmöglichkeiten entstanden, das behinderten psychisch Kranken ermöglicht, ausserhalb der psychiatrischen Krankenhäuser zu leben. Im Vergleich mit anderen Ländern fehlt es aber nunmehr mit ganz wenigen Ausnahmen an indivi- 
duell geförderter Integration auf dem allgemeinen Arbeitsmarkt («Supported Employment», «Job Coach»). Darüber hinaus sind in den vergangenen Jahren zusätzlich eine Reihe personenzentrierter rehabilitativer Verfahren und Strategien entwickelt worden, die wirksame Behandlungsmethoden darstellen. Zum einen richten sich dabei die Behandlungsstrategien auf die psychischen Funktionseinschränkungen, die in Störungen der Merkfähigkeit, der Konzentration, der Gefühle, der Stimmung, der Motivation, der Ausdauer, der Belastbarkeit, des Antriebs oder auch in einer eingeschränkten Kritik- und Urteilsfähigkeit ihren Ausdruck finden. Zum anderen zielen diese Rehabilitationsansätze auf die soziale Behinderung, wie z.B. auf eine eingeschränkte Kommunikations- und Kontaktfähigkeit, auf eine unzureichende Selbstversorgung oder auf die mangelhafte Bewältigung alläglicher Aufgaben ab Störungsbereiche, die sich aus den vorgenannten seelischen Funktionseinschränkungen entwickeln.

Grosse Popularität haben z. B. in den vergangen Jahren computergestützte Rehabilitationsprogramme für neuropsychologische Defizite erlangt. Auch persistierende Wahnideen und Halluzinationen können mit psychologischen Therapieansätzen, die den Betroffenen funktionsangepasste «neue Erklärungen» für die Inhalte der Wahngedanken und Halluzinationen geben, erfolgreich angegangen werden. Andere Interventionsmethoden zielen darauf, soziale Fertigkeiten und Kompetenzen zu vermitteln. Dabei geht es im Kern um ein Training grundlegender Fertigkeiten zur Bewältigung des Alltagslebens. Die Resultate verschiedener kontrollierter Studien zeigen, dass den Betroffenen mit diesen Lernprogrammen ein weites Spektrum unterschiedlicher sozialer Fertigkeiten vermittelt werden kann. Über diese psychotherapeutischen Ansätze hinaus wurden ausserdem verschiedene psychoedukative Programme für eine bessere Krankheitsbewältigung entwickelt. Ausgearbeitete Programme existieren z. B. im Hinblick auf die Früherkennung von Rückfällen. Die wichtigsten edukativen Programme sind aber im Bereich der Angehörigenarbeit ausgearbeitet worden. Familienprogramme sind in mehrfacher Hinsicht wirksam. Sie reduzieren die Rückfallrate, verbessern das soziale Funktionsniveau der Betroffenen und können auch die familiäre Belastung reduzieren. Im Bereich der beruflichen Reintegration schliesslich haben einschlägige Untersuchungen konsistent gezeigt, dass «Supported Employment»-Programme, bei denen eine rasch Plazierung an einen regulären Arbeitsplatz mit begleitender intensiver Betreuung vor Ort erfolgt («zuerst plazieren und dann trai- nieren»), den herkömmlichen Integrationsversuchen mit vorgängigem Arbeitstraining in geschütztem Rahmen («zuerst trainieren und dann plazieren») klar überlegen sind.

Auch die moderne Rehabilitationspraxis ist also mit einem Perspektivenwechsel seelischer Erkrankungen verbunden: Im Zentrum steht ein Modell funktionaler Beeinträchtigung, d.h., der Verlauf der Erkrankung wird nicht mehr vorrangig oder allein an der Krankheitssymptomatik gemessen, sondern an der Fähigkeit des Betroffenen, seine sozialen Rollen in Familie, Arbeit und gesellschaftlichem Leben zu erfüllen. Dass entsprechende Trainings- und Behandlungsprogramme wirksamer sind, wenn sie im natürlichen Lebensumfeld statt in einem stationären Setting zum Einsatz kommen, ist deshalb nicht überraschend. Integration in Beruf und Gesellschaft hängt aber nicht nur von den Bemühungen der Betroffenen und ihrer Helfer ab, sondern auch von der Bereitschaft der Gesellschaft, ihre beeinträchtigten Mitglieder zu integrieren. Die Haltung der Bevölkerung zu psychisch Kranken bestimmt das Gelingen der Reintegration, indem sie das Lebensumfeld prägt, in das ein psychisch Kranker zurückkehrt bzw. das er sich neu erschliessen muss. Die Akzeptanz bzw. Ablehnung der direkten sozialen Umwelt hat somit einen nicht unwesentlichen Einfluss auf das Ergebnis der Rehabilitation. Oft genug internalisieren die Betroffenen das Stigma ihrer Erkrankung. Selbstwertzweifel führen sie in Isolation und Rückzug. Diesem Prozess der kontinuierlichen Entwertung haben im vergangenen Jahrzehnt Angehörige und Betroffene ein Modell der Ermutigung entgegengesetzt. Für die professionellen Helfer ist es wichtig zu akzeptieren, dass Betroffene und Angehörige Experten ihrer eigenen Befindlichkeit sind. Neben dem Respekt vor der subjektiven Krankheitseinsicht der Betroffenen, der Betonung ihrer vorhandenen Fähigkeiten wie auch dem absoluten Primat der Freiwilligkeit der Behandlung, ist es vor allen Dingen die therapeutische Allianz zwischen Patient und Therapeut, die den Rahmen für eine erfolgreiche Rehabilitation abgibt.

Der im internationalen Vergleich hohe Bettenschlüssel der Schweiz ist also auch wesentlich mit darauf zurückzuführen, dass die heutigen Möglichkeiten einer gemeindeintegrierten Rehabilitation bisher in noch viel zu geringem Umfang genutzt werden. So verbleiben zu viele chronisch Kranke zu lange in stationärer Behandlung, weil insbesondere die erforderlichen Einrichtungen für das betreute Wohnen nicht verfügbar sind. Diese müssen vom individuellen Wohnen bis hin zu intensiv betreuten Wohn- 
einheiten reichen («ward in a house», «hospital hostel»). Es ist deshalb mehr als nur kurzsichtig, wenn in letzter Zeit unter dem Druck begrenzter Psychiatriebudgets einzelne Rehabilitationsstationen geschlossen werden, ohne einen Teil der damit frei werdenden Ressourcen in gemeindeintegrierte Behandlungs- und Betreuungsformen zu transferieren. Auf diese Weise wird sich nur der Druck auf die Akutstationen weiter erhöhen und ein Teil der dort notgedrungen versorgten Patienten wird statt dem billigeren Rehabilitationsbett das teurere, für eine weitere Behandlung aber ungeeignete Akutbett belegen.

\section{Interdisziplinarität und Multiprofessionalität psychiatrischer Behandlungsdienste}

An sich besteht allgemeiner Konsens darüber, dass es verbindlicher Standard ist, die psychiatrische Versorgung am bio-psycho-sozialen Krankheitsmodell zu orientieren. Dabei ist von entscheidender Bedeutung, dass psychische Erkrankungen sich im biologischen Bereich, im subjektiven Erleben und im sozialen Verhalten manifestieren und über diese drei Ebenen sowie über deren Wechselwirkungen zum Positiven wie zum Negativen beeinflussbar sind. Dieser ganzheitliche Ansatz erfordert die enge Verzahnung der ärztlichen, pflegerischen, psycho-, ergo- und physiotherapeutischen Behandlung und Betreuung unter Einschluss von Hilfen zur Bewältigung psychosozialer Krisen- und Problemsituationen. In der stationären Akut- wie Rehabilitationsbehandlung - wobei frühe rehabilitative Leistungen mehr und mehr Bestandteil auch der akuten Behandlung sind - ist dies seit langem eine Selbstverständlichkeit. Medizinische Leistungen der Krankenversicherung umfassen hier durchaus auch psychosoziale Leistungen im weiteren Sinn, wie zum Beispiel die Kontaktaufnahme mit Sozialdiensten und Behörden, Hilfe bei der Wohnungssuche, Kontaktaufnahme mit den Arbeitgebern, Schuldnerberatung usw. Es würde zu Recht als widersinnig empfunden, diese Leistungen aus den Tagesoder Fallpauschalen herauszurechnen. Vielmehr werden sie richtigerweise im Sinne eines Komplexleistungsprogramms als integraler Bestandteil der stationären Behandlung betrachtet. Dies macht auch ökonomisch Sinn, denn Leistungen zur Selbstversorgung, zur Tagesgestaltung, zur Kontaktfindung usw. sind notwendig, um zum Beispiel psychische Funktionen wie Motivation, Antrieb, Konzentration oder Selbstvertrauen zu stärken, um Verschlechterungen des Gesund- heitszustandes zu verhüten oder um Pflegebedürftigkeit zu verhindern.

Nun ist es aber - wie oben im einzelnen aufgezeigt - weder vom wissenschaftlichen Stand der Erkenntnis über Wirksamkeit und KostenNutzen-Relation noch von den Erfahrungen in der Versorgungspraxis her vertretbar, dass diese Komplexleistungen nur im Krankenhaus erbracht werden sollen. Die bisherige finanzielle Schlechterstellung einer entsprechenden multiprofessionellen Behandlung im ambulanten Feld kann weder mit wissenschaftlichen Argumenten noch mit Gründen der Wirksamkeit, Zweckmässigkeit oder Wirtschaftlichkeit begründet werden, da ambulante Versorgungsstrukturen, welche solche multiprofessionellen therapeutischen Angebote gebündelt vorhalten und im Lebensbereich des Patienten bedarfsgerecht zur gezielten Anwendung bringen, nicht nur ausreichend erprobt, sondern auch kosteneffizient sind.

Im Zentrum eines modernen institutionellen psychiatrischen Versorgungssystems muss heute deshalb die ambulante psychiatrische Grundversorgung durch ein multiprofessionelles Team stehen, in die bedarfsorientiert und personenzentriert spezielle Therapieverfahren und Leistungen zur Selbstversorgung, zur Hilfe bei der Arbeit und Ausbildung, bei der Tagesgestaltung und Kontaktfindung sowie die teilstationäre und stationäre Behandlung und erforderlichenfalls ergänzende nicht psychiatrische medizinische Leistungen integriert werden. Bei der ambulanten psychiatrischen Grundversorgung handelt es sich also nicht lediglich um additiv zusammengefügte, sondern um stringent aufeinander abgestimmte Hilfen verschiedener Fachpersonen unter ärztlicher Leitung. Dies gilt in völlig analogem Sinn wie bei der stationären Versorgung, bei der es sich leistungsrechtlich trotz der Vielzahl von Einzelmassnahmen, die sie umfasst, um eine einzige Leistung mit der Zuständigkeit eines Leistungsträgers handelt. Welchen Inhalt und Umfang die dabei konzeptionell, organisatorisch und personell verbundenen einzelnen Hilfen haben, muss sich nach den Erfordernissen im Einzelfall richten.

\section{Abgeltungssysteme und tarifliche Regelungen}

In der Schweiz regelt das seit dem 1. Januar 1996 in Kraft stehende «Bundesgesetz über die Krankenversicherung (KVG)» die Leistungen, welche durch die obligatorische Krankenversicherung bei Krankheit, Unfall und Mutterschaft über- 
nommen werden. Art. 2 KVG hält dazu fest: «Krankheit ist jede Beeinträchtigung der körperlichen oder geistigen Gesundheit, die nicht Folge eines Unfalles ist und die eine medizinische Untersuchung oder Behandlung erfordert oder eine Arbeitsunfähigkeit zur Folge hat. Unfall ist die plötzliche, nicht beabsichtigte schädigende Einwirkung eines ungewöhnlichen äusseren Faktors auf den menschlichen Körper, die eine Beeinträchtigung der körperlichen oder seelischen Gesundheit zur Folge hat.» Krankheitswertige psychische Störungen inkl. Veränderungen der seelischen Gesundheit in der Folge von Unfällen oder in Zusammenhang mit Schwangerschaft oder Niederkunft fallen beispielsweise eindeutig in diese Kategorie. Zum Leistungsumfang heisst es im KVG, dass die obligatorische Krankenversicherung die Kosten für diejenigen Leistungen übernimmt, die der Diagnose oder der Behandlung einer Krankheit und ihrer Folgen dienen (Art. 25, 1). Unfälle sind bezüglich Leistungen Krankheiten gleichgestellt (Art. 28). Diese Leistungen umfassen die Untersuchungen, Behandlungen und Pflegemassnahmen, die ambulant, bei Hausbesuchen, stationär, teilstationär oder in einem Pflegeheim von Ärzten oder Ärztinnen oder von Personen, die auf Anordnung oder im Auftrag eines Arztes oder einer Ärztin Leistungen erbringen (Art. 25, 2 a). Sie müssen wirksam, zweckmässig und wirtschaftlich sein; die Wirksamkeit muss nach wissenschaftlichen Methoden nachgewiesen werden (Art. 32).

In den vorangegangenen Abschnitten wurde aufgezeigt, wie die moderne psychiatrische Versorgung heute ausgerichtet sein sollte. Es wurde dargestellt, dass die interdisziplinäre (mehrere Fachleute sind für einen kranken Menschen engagiert), pluriprofessionelle (diese Fachleute gehören verschiedenen Berufsgruppen an) Arbeit im ambulanten, teilstationären und stationären Bereich heute Standard ist. Dank dieser «integrierten psychiatrischen Behandlung», die biologische, psychologische und soziale Aspekte gleichwertig einbezieht und berücksichtigt, können heute psychisch Kranke zunehmend erfolgreich behandelt und medizinisch rehabilitiert werden (Art. 25, d). Ihre Wirksamkeit und Zweckmässigkeit speziell auch im gemeindeintegrierten Feld sind wissenschaftlich untermauert und auch ihre Wirtschaftlichkeit ist gegeben, da damit erfolgreich behandelte Kranke weniger lang und weniger intensiv Leistungen aus den Sozialversicherungen beziehen. Sie sind früher im Berufs- und Erwerbsleben wieder tätig, wodurch auch volkswirtschaftlich gesehen die Kosten kleiner gehalten werden können.
Im Rahmen der Verhandlungen um den zukünftigen Tarif TARMED konnten im Kapitel «Nichtärztliche Leistungen in der Psychiatrie» im Unterkapitel 02.04 Tarifpositionen definiert und eingefügt werden, die in Anlehnung an die erwähnten Artikel des Krankenversicherungsgesetzes KVG zukünftig als Grundlagen für die Verrechnungen der beschriebenen modernen Behandlungsverfahren dienen können. Damit ist zunächst einmal zumindest im Grundsatz sichergestellt, dass endlich auch in der Schweiz die Weiterentwicklung des psychiatrischen Versorgungssystems nach den Prinzipien der Gemeindeintegration, Patientenzentrierung und Ambulantisierung in Gang kommen kann. Noch nicht gelöst sind aber die Probleme, die man unter dem Stichwort «Anreizsysteme» zusammenfassen kann. Geld steuert Verhalten von Gesunden ebenso wie von Kranken in entscheidenden Aspekten. Hier sind deshalb neue Denkund Abgeltungsmodelle im KVG- und IVG-Bereich bei der Behandlung sowohl psychisch akut Erkrankter wie psychisch Behinderter und bei ihrer Betreuung und Wiedereingliederung in die Gesellschafts- und Arbeitswelt erforderlich. Diese beiden Sozialversicherungen spielen hier eine zentrale Rolle und bestimmen ganz wesentlich mit, wie das psychiatrische Versorgungssystem aussieht und sich weiterentwickeln wird, und ob sich wissenschaftlich gebotene innovative Versorgungsstrukturen und -konzepte in der Versorgungspraxis auch tatsächlich etablieren lassen. Dazu gibt es aus anderen Ländern eindrückliche Beispiele. So führte etwa die zeitlich befristete tarifliche Gleichstellung von Tagesklinikplätzen mit Klinikbetten in einigen bayerischen Regierungsbezirken rasch zu einer psychiatriepolitisch wünschenswerten Umschichtung von stationärer zu teilstationärer Behandlung, von der dann auch die Leistungsträger profitierten. Ein anderes Beispiel lässt sich aus Nordrhein-Westfalen anführen, wo die Pauschalvergütung für die integrierte psychiatrische Behandlung durch Klinikteams, die sowohl Behandlung im Hause des Patienten, teilstationäre Behandlung wie - soweit unumgänglich - stationäre Behandlung anbieten, entsprechend den Tagessätzen für vollstationäre Patienten im Umfang der durchschnittlichen Aufenthaltsdauer an der jeweiligen Klinik erfolgt. Auch hier profitieren nach den Ergebnissen der Begleitforschung letztlich nicht nur Patienten und ihre Angehörigen von der damit verbundenen Versorgungsinnovation, sondern wiederum auch die Leistungsträger. Ein weiteres Beispiel stellt der in einigen deutschen Bundesländern derzeit 
modellhaft erprobte Übergang von der Objektzur Subjektfinanzierung dar, wobei personenzentrierte, individuelle Komplexleistungsprogramme im natürlichen Lebensumfeld des Patienten ermöglicht werden, die sich zudem flexibel an den sich im Krankheitsverlauf verändernden Hilfebedarf anpassen lassen. Auch wenn die Sozialversicherungssysteme in der Schweiz an sich hervorragend sind, müssen sie zur Förderung von gemeindeintegrierter Krisenintervention und Akutbehandlung sowie zur rehabilitationsorientierten Behandlung, Betreuung und Wiedereingliederung psychisch Kranker und Behinderter doch im Verordnungs- und Vertragsbereich noch optimiert werden.

\section{Psychiatriepolitische Erfordernisse}

Die hier aufgezeigte Umorientierung des institutionellen psychiatrischen Versorgungssystems wird nicht ohne entsprechende psychiatriepolitische Vorgaben erfolgen können, zumal von der Psychiatrie schon ganz grundsätzlich im Vergleich mit anderen Bereichen der Medizin einige für das politische Handeln relevante Besonderheiten bestehen. Die politische Aufgabenstellung ist hier umfassender, weil Prävention psychischer Störungen und die Diagnostik und Behandlung psychischer Erkrankungen sämtliche zentralen Lebensbereiche wie Wohnen, Familie, Arbeit usw. in direkterer Weise betreffen, als dies bei somatischen Erkrankungen in der Regel der Fall ist. Diese ausgeprägteren sozialen Auswirkungen führen häufiger zu Konflikten mit anderen gesellschaftlichen und politischen Interessen. Psychiatriepolitik muss deshalb gleichzeitig die Anliegen der psychisch Kranken und ihrer Angehörigen verfolgen, wie auch das gesellschaft liche Ganze berücksichtigen, d.h. überprüfen, welche Auswirkungen bestimmte psychiatriepolitische Massnahmen für die Gesellschaft insgesamt haben, und wie weit die Auswirkungen für die Bürger überhaupt akzeptabel sind. Daraus ergeben sich Fragen, die nicht alleine oder nicht in erster Linie durch das Fachwissen von Experten, sondern nur mittels Wertsetzungen und Zielvorgaben der verantwortlichen Politiker beantwortbar sind. Zur Ingangsetzung des notwendigen Planungsprozesses bedarf es einer Psychiatriekommission oder eines Psychiatriebeirats, in dem neben den Leistungsträgern und den Leistungserbringern auch Psychiatrieerfahrene und Angehörige vertreten sind. Dieses Gremium soll über den vorhandenen Entwicklungsbedarf, die Planungsgrundsätze sowie Umsetzungsfragen beraten. Es soll die Verständi- gung zwischen allen beteiligten Gruppen über grundsätzliche konzeptionelle, institutionelle und finanzielle Fragen anstreben, deren Klärung für die Ingangsetzung von Einzelvorhaben notwendig ist. Hinsichtlich der Versorgungserfordernisse und ihrer Bewältigung soll es Empfehlungen an die politischen Entscheidungsträger formulieren. Entsprechend den herkömmlichen Planungsverfahren können dabei Messziffern pro Einrichtungstyp verwendet und auf die Bevölkerung projiziert werden. Dieser Ansatz ist einfach rechnerisch umsetzbar und bewirkt in Verbindung mit der räumlich definierten Betten/ Platzzahl eine Angebotsbegrenzung. Eine zukünftige Orientierung an einem wünschenswerten personenzentrierten Ansatz, bei dem sich die Entwicklung von regionalen Hilfefunktionen am konkreten Hilfebedarf der in der Region lebenden Personen ausrichtet, ist auf dieser Grundlage möglich.

\section{Schlussbemerkungen}

Abschliessend sei warnend darauf hingewiesen, dass speziell die ungenügende Anpassung leistungsrechtlicher Bestimmungen an den Stand der internationalen Versorgungsforschung sowie das Fehlen modellhafter tariflicher Anreizsysteme für eine gemeindeintegrierte und personenzentrierte Behandlung und Betreuung in der Schweiz gegenwärtig eine fachlich wie wirtschaftlich gesehen völlig kontraproduktive Rückverlagerung psychiatrischer Leistungen in die Klinik begünstigt, die zu Lasten aller Beteiligten geht. Die zum Ausgangspunkt der hier angestellten Überlegungen genommene Steigerung der Aufnahmezahlen in stationäre Behandlung ist nicht zuletzt auch unter diesem Aspekt $\mathrm{zu}$ sehen. Je mehr die Balance der verfügbaren Ressourcen zwischen gemeindeintegrierter Krisenintervention und Akutbehandlung, ambulanter Nachbehandlung, Betreuung und Rehabilitation einerseits und stationärer Behandlung andererseits zu Ungunsten der ambulanten und teilstationären Hilfsangebote verschoben wird, um so mehr wird der Finanzbedarf in der stationären Behandlung zunehmen und schnell einmal die Höhe des heute verfügbaren Gesamtbudgets erreichen. An Stelle der kurzschlüssigen Eröffnung neuer Akutbetten wäre deshalb die Stärkung der gemeindeintegrierten Behandlungsangebote die wirksamere, nachhaltigere und auch kosteneffizientere Steuerungsmassnahme. Alle an der institutionellen psychiatrischen Versorgung beteiligten Gruppen sind zu entsprechendem Handeln aufgerufen. 


\section{Literatur}

1 Stein LJ, Test MA. Alternative to mental hospital treatment. 1. Conceptual model treatment program and clinical evaluation. Arch Gen Psychiatry 1980;37:392-7.

2 Burns BJ, Santos AB. Assertive community treatment: an update of randomized trials. Psychiatr Serv 1995;46:669-75.

3 Mueser KT, Bond GR, Drake RE, Resnick SG. Models of community care for severe mental illness: a review of research on case management. Schizophr Bull 1998;24:37-74.

4 Brenner HD, Junghan U, Pfammatter M. Gemeindeintegrierte Akutversorgung Möglichkeiten und Grenzen. Nervenarzt 2000; 71:691-9.

5 Creed F, Black D, Anthony P, Osborn M, Thomas P, Tomenson B. Randomised controlled trial of day patient versus inpatient psychiatric treatment. Br Med J 1990;300:1033-7.

6 Merson S, Tyrer P, Onyett S, Lack S, Birkett P, Lynch S, Johnson T. Early intervention in psychiatric emergencies: a controlled clinical trial. Lancet 1992;339:1311-4.

7 Burns T, Beadsmoore A, Bhat AV, Oliver A, Mathers C. A controlled trial of home-based acute psychiatric services. I: clinical and social outcome. Br J Psychiatry 1993;163:49-54.

8 Nienhuis FJ, Giel R, Kluiter H, Rüphan M, Wiersma D. Efficacy of psychiatric day treatment. Course and outcome of psychiatric disorders in a randomised trial. Eur Arch Psychiatry Clin Neurosci 1994;244:73-80.

9 Marks IM, Connolly J, Muijen M, Audini B, McNamee G, Lawrence RE. Home-based versus hospital-based care for people with serious mental illness. Br J Psychiatry 1994;164:179-94.

10 Schene AH, van Wijngaarden B, Poelijoe NW, Gersons BPR. The Utrecht comparative study on psychiatric day treatment and inpatient treatment. Acta Psychiatr Scand 1993;87:427-43.

11 Sledge WH, Tebes J, Rakfeldt J, Davidson L, Lions L, Druss B. Day hospital/crisis respite care versus inpatient care, part I: clinical outcome. Am J Psychiatry 1996;153:1065-73.
12 Burns T, Raftery J, Beadsmoore A, McGuigan S, Dickson M. A controlled trial of home-based acute psychiatric services. II: treatment patterns and costs. Br J Psychiatry 1993;163:55-61.

13 Merson S, Tyrer P, Carlen D, Johnson T. The cost of treatment of psychiatric emergencies: a comparison of hospital and community services. Psychol Med 1996;26:727-34.

14 Creed F, Mbaya P, Lancashire S, Tomenson B, Williams B, Holme S. Cost-effectiness of day and inpatient psychiatric treatment: results of a randomised controlled trial. Br Med J 1997; 314:1381-5.

15 Sledge WH, Tebes J, Wolff N, Helminiak TH. Day hospital/crisis respite care versus inpatient care, part II: service utilization and costs. Am J Psychiatry 1996;153:1074-83.

16 Knapp M, Beecham J, Koutsogeorgopoulou V, Hallam A, Fenyo A, Marks IM, et al. Service use and costs of home-based versus hospital-based care for people with serious mental illness. Br J Psychiatry 1994;165:195-203.

17 Wiersma D, Kluiter H, Nienhuis FJ, Rüphan M, Giel R. Costs and benefits of hospital and day treatment with community care of affective and schizophrenic disorders. Br J Psychiatry 1995; 166(suppl 27):52-9.

18 Wykes T. The toxicity of community care. In: Tyrer P, Creed F (eds.). Community Psychiatry in Action. Analysis and Prospects. Cambridge: University Press; 1995. p. 97-111.

19 Kluiter H. Inpatient treatment and care arrangements to replace or avoid it - searching for an evidence-based balance. Curr Opin Psychiatry 1997;10:160-7.

20 Davidson L, Tebes JK, Rakfeldt J, Sledge W. Differences in social environment between inpatient and day hospital-crisis respite settings. Psychiatr Serv 1996;47:714-20.

21 Thornicroft G, Wykes T, Holloway F, Johnson S, Szmukler G. From efficacy to effectiveness in community mental health services. PRiSM Psychosis Study 10. Br J Psychiatry 1998; 173:423-42. 